\title{
Translocation of molecular chaperones to the titin springs is common in skeletal myopathy patients and affects sarcomere function
}

Andreas Unger ${ }^{1,2}$, Lisa Beckendorf ${ }^{1}$, Pierre Böhme ${ }^{3}$, Rudolf Kley ${ }^{3}$, Marion von Frieling-Salewsky ${ }^{4}$, Hanns Lochmüller ${ }^{5}$, Rolf Schröder ${ }^{6}$, Dieter O. Fürst ${ }^{7}$, Matthias Vorgerd ${ }^{3}$ and Wolfgang A. Linke ${ }^{1,4^{*}}$

\begin{abstract}
Summary: Myopathies encompass a wide variety of acquired and hereditary disorders. The pathomechanisms include structural and functional changes affecting, e.g., myofiber metabolism and contractile properties. In this study, we observed increased passive tension (PT) of skinned myofibers from patients with myofibrillar myopathy (MFM) caused by FLNC mutations (MFM-filaminopathy) and limb-girdle muscular dystrophy type-2A due to CAPN3 mutations (LGMD2A), compared to healthy control myofibers. Because the giant protein titin determines myofiber PT, we measured its molecular size and the titin-to-myosin ratio, but found no differences between myopathies and controls. All-titin phosphorylation and site-specific phosphorylation in the PEVK region were reduced in myopathy, which would be predicted to lower PT. Electron microscopy revealed extensive ultrastructural changes in myofibers of various hereditary myopathies and also suggested massive binding of proteins to the sarcomeric I-band region, presumably heat shock proteins (HSPs), which can translocate to elastic titin under stress conditions. Correlative immunofluorescence and immunoelectron microscopy showed that two small HSPs (HSP27 and aB-crystallin) and the ATP-dependent chaperone HSP90 translocated to the titin springs in myopathy. The small HSPs, but not HSP90, were upregulated in myopathic versus control muscles. The titin-binding pattern of chaperones was regularly observed in Duchenne muscular dystrophy (DMD), LGMD2A, MFM-filaminopathy, MFM-myotilinopathy, titinopathy, and inclusion body myopathy due to mutations in valosin-containing protein, but not in acquired sporadic inclusion body myositis. The three HSPs also associated with elastic titin in mouse models of DMD and MFM-filaminopathy. Mechanical measurements on skinned human myofibers incubated with exogenous small HSPs suggested that the elevated PT seen in myopathy is caused, in part, by chaperone-binding to the titin springs. Whereas this interaction may be protective in that it prevents sarcomeric protein aggregation, it also has detrimental effects on sarcomere function. Thus, we identified a novel pathological phenomenon common to many hereditary muscle disorders, which involves sarcomeric alterations.
\end{abstract}

Keywords: Myofibrillar myopathy, Muscular dystrophy, Muscle stiffness, Molecular chaperones, Immunoelectron microscopy

\footnotetext{
*Correspondence: wlinke@uni-muenster.de

'Department of Cardiovascular Physiology, Ruhr University Bochum, Bochum, Germany

${ }^{4}$ Institute of Physiology II, University of Muenster, Robert-Koch-Str. 27b, 48149

Münster, Germany

Full list of author information is available at the end of the article
}

(c) The Author(s). 2017 Open Access This article is distributed under the terms of the Creative Commons Attribution 4.0 International License (http://creativecommons.org/licenses/by/4.0/), which permits unrestricted use, distribution, and reproduction in any medium, provided you give appropriate credit to the original author(s) and the source, provide a link to the Creative Commons license, and indicate if changes were made. The Creative Commons Public Domain Dedication waiver (http://creativecommons.org/publicdomain/zero/1.0/) applies to the data made available in this article, unless otherwise stated. 


\section{Introduction}

Hereditary myopathies are a clinically and genetically heterogeneous group of disorders with a variable age of onset, from congenital to late adulthood. The myopathies have been classified into subgroups based on the clinical distribution of muscle weakness (e.g. proximal vs. distal) and the inheritance pattern (e.g. autosomal dominant vs. recessive), before the genetic etiology was revealed [43]. Today, mutations in dozens of different genes are known to cause a myopathy [27], with Duchenne muscular dystrophy (DMD) due to mutations in dystrophin being the most frequent. A large subgroup encompasses the limbgirdle muscular dystrophies (LGMDs) caused by mutations in at least 30 different genes with autosomal dominant (LGMD1; 9 genes/loci) or autosomal recessive (LGMD2; 24 genes) inheritance [57]. One of the most frequent subtypes of LGMD is LGMD2A caused by homozygous or compound heterozygous mutations in the gene encoding the proteolytic enzyme calpain-3 (CAPN3), a known interactor of the giant sarcomere protein titin $[16,48]$. Myofibrillar myopathies (MFMs), another group of hereditary muscle disorders, are characterized by histological features including focal disintegration of myofibrils and protein aggregation in myofibers. Known MFM disease genes encode proteins associated with the sarcomeric Z-disc, such as myotilin, desmin, and filamin-C [29]. For many LGMDs and MFMs, the molecular mechanisms underlying the respective disorder remain largely unresolved and specific or ameliorating therapies are not available.

In our previous approaches to this topic, we focused mainly on mechanisms of protein quality control, which we found to be pathologically altered in MFMs [30]. We deciphered distinct mutation-specific disease mechanisms in the human myopathies, such as protein misfolding and aggregation, toxic gain of function, and haploinsufficiency [2, 21]. Recently, we demonstrated that the small heat shock proteins (sHSPs), HSP27 (HSPB1) and $\alpha$ B-crystallin (HSPB5), which in healthy muscle cells localize to the Z-disc or cytosol, were translocated to the titin springs of the sarcomeric I-bands in LGMD2A myocytes [31]. Titin is established as being responsible for the elasticity and "passive" tension (PT) of the myocyte [39], but is also evolving as a protein critical for the active mechanical properties of the sarcomere $[26,36,52]$. The binding of HSPs to titin suggested a role for these molecular chaperones in the pathomechanism of myopathy subtypes, which are presenting with reduced contractile force generation and increased muscle stiffness.

HSPs are important components of protein quality control, as they affect protein folding and promote degradation, e.g. via the ubiquitin-proteasome system (UPS) or autophagy pathways $[7,56]$. Members of the family of sHSPs assist in the folding and maintenance of the cytoplasmic proteome and are considered holdases rather than foldases [5, 42]. Interestingly, overexpression of sHSPs significantly reduces aberrant protein aggregation in cell and animal models of MFM [10, 53, 54, 59]. Moreover, chemical chaperones can impede pathological protein aggregation and improve muscle function [60]. We found that sHSPs stabilize folded immunoglobulinlike (Ig) domains of titin from the elastic I-band segment [9]. If these Ig modules unfold in response to sarcomere stretching [1, 52], the sHSPs may help protect them from aggregation [31]. The ATP-dependent chaperone HSP90 is known to assist in the assembly of the myosin filaments [55] and alter myosin motor function [47]. HSP90 also binds, if methylated by the methyltransferase Smyd2, to the N2A element of I-band titin (near the calpain-3-binding site) and exerts a protective effect on Z-disc/I-band structure [15, 58]. HSP90 is among the chaperones discussed as potential ameliorators of dystrophic muscle disease [8]. However, its relevance in LGMDs and MFMs has not been studied.

Considering the potential for chaperones to improve myocyte function in muscle disease, we initiated this study with the aim to better understand the relationship between titin and HSPs in human hereditary myopathies. We set out to determine which chaperones associate with titin in muscle biopsies from different myopathies, including LGMD2A and MFM-filaminopathy. We found that, of all HSPs studied, only HSP27, $\alpha \mathrm{B}$-crystallin and HSP90 were translocated from the cytosol or sarcomeric Z-disc in healthy human muscles to the titin springs in myopathy. We mapped the interaction sites by immunoelectron microscopy and measured the impact of endogenous HSP-binding to the sarcomeres on myofiber PT, in controls and myopathy patients. We also tested whether exogenous HSPs added to permeabilized human myofibers affect PT. By examining biopsy material from control subjects, 17 patients with different myopathies, and muscle from animal models of hereditary myopathies, we found that massive HSP-binding to titin is a common feature in dystrophic and MFM muscle disorders. We conclude that the translocation of HSPs to titin, while protecting the protein in the sarcomeres, could also impair titin-based myofiber elasticity, presumably contributing to increased muscle stiffness. These alterations represent a previously unrecognized pathophenomenon in hereditary myopathies.

\section{Methods}

\section{Human muscle biopsies}

We studied M. vastus lateralis and gastrocnemius biopsies from three healthy (CTRL) subjects with normal histopathological features and 17 diseased human subjects with various muscle disorders (see Table 1). The following hereditary myopathies were included (mutated 
Table 1 Overview of human and mouse muscle samples studied and intracellular localization of major chaperones

\begin{tabular}{|c|c|c|c|c|c|}
\hline \multirow{2}{*}{ Gene } & \multirow[b]{2}{*}{ Disease/Mutation } & \multirow[b]{2}{*}{ Year of birth/Gender } & \multicolumn{3}{|c|}{ Intracellular localization of } \\
\hline & & & aB-Crystallin & HSP27 & HSP90 \\
\hline \multicolumn{6}{|c|}{ Human muscles } \\
\hline- & (3x) Healthy CTRL & 1953/72/78/M & Z-disc & Z-disc/Cytosol & Cytosol \\
\hline \multirow[t]{4}{*}{ CAPN3 } & 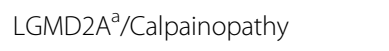 & & I-band & I-band & I-band \\
\hline & 1) p.Thr184Argfs \& Trp130 > Cys & 1967/M & & & \\
\hline & 2) p.Thr184Argfs \& p.Arg315Arg & 1957/M & & & \\
\hline & 3) p.Thr184Argfs \& p.Gly329Arg & $1968 / F$ & & & \\
\hline \multirow[t]{3}{*}{$D M D$} & Duchenne muscular dystrophy & & I-band & I-band & I-band \\
\hline & 1) Del. exon 2-18 & 1998/M & & & \\
\hline & 2) Del. exon 3-11 & 2014/M & & & \\
\hline FLNC & $\begin{array}{l}\text { Filaminopathy } \\
\text { (2x) p.Val930_Thr/933del }\end{array}$ & $\begin{array}{l}\text { 1960/M } \\
\text { 1960/F }\end{array}$ & I-band & I-band & I-band \\
\hline TTN & $\begin{array}{l}\text { Titinopathy/HMERF } \\
(2 x) \text { p.Cys30071Arg }\end{array}$ & Unknown/Unknown & I-band & I-band & I-band \\
\hline \multirow[t]{3}{*}{ MYOT } & Myotilinopathy & & I-band & I-band & I-band \\
\hline & 1) p.Ser60Phe & 1928/M & & & \\
\hline & 2) p.Lys36Glu & 1940/F & & & \\
\hline \multirow[t]{3}{*}{ VCP } & $\begin{array}{l}\text { IBMPFDC }^{c} \\
\text { (Valosin-containing protein) }\end{array}$ & & I-band & I-band & I-band \\
\hline & 1) p.Arg93Cys & 1971/M & & & \\
\hline & 2) p.Arg155His & Unknown/M & & & \\
\hline DES & $\begin{array}{l}\text { Desminopathy } \\
\text { p.Arg350Pro }\end{array}$ & 1976/M & Cytosol & Cytosol & I-band/ Cytosol \\
\hline- & $\begin{array}{l}\text { ( } 3 \times) \text { Sporadic inclusion body } \\
\text { myositis (sIBM) }\end{array}$ & 1955/1998/2014/M & Z-disc & Z-disc/Cytosol & Cytosol \\
\hline \multicolumn{6}{|c|}{ Mouse muscles } \\
\hline- & Litter-matched WT CONTROLS & 2016/M & Z-disc & Z-disc/Cytosol & Cytosol \\
\hline FLNC & $\begin{array}{l}\text { MFM filaminopathy } \\
\text { p.W2711X }\end{array}$ & 2016/M & I-band & I-band & I-band \\
\hline$D M D$ & $\mathrm{mdx}$ C57BL/10ScSn & 2016/M & I-band & I-band & I-band \\
\hline
\end{tabular}

aGMD2A, Limb girdle muscular dystrophy type $2 \mathrm{~A}$

${ }^{\mathrm{b}} \mathrm{HMERF}$, Hereditary myopathy with early respiratory failure

IBMPFD, Inclusion body myopathy with Paget disease and frontotemporal dementia

gene and specific mutation(s) listed in parentheses): Among the group of muscular dystrophies, LGMD2A (CAPN3; p.Thr184Argfs \& Trp130 > Cys, p.Thr184Argfs \& p.Arg315Arg, p.Thr184Argfs \& p.Gly329Arg) and Duchenne muscular dystrophy ( $D M D$; Del. exon 2-18, Del. exon 3-11); among the group of myofibrillar myopathies, filaminopathy (FLNC; p.Val930_Thr/933del $(2 \times))$, desminopathy (DES; p.Arg350Pro), myotilinopathy (MYOT; p.Ser60Phe, p.Lys36Glu), and a titinopathy, hereditary myopathy with early respiratory failure (HMERF) (TTN; p.Cys30071Arg (2x)); hereditary inclusion body myopathy (inclusion body myopathy with Paget disease and frontotemporal dementia, IBMPFD) caused by valosin-containing protein mutation (VCP; p.Arg93Cys, p.Arg155His). At least two biopsy samples per disorder (in filaminopathy from two siblings) were analyzed, with the exception of desminopathy, from which only a single biopsy sample was available. Additionally, we included biopsies from three patients with acquired sporadic inclusion body myositis.

\section{Ethics, consent and permissions}

Patients consented to participate in this study, which conforms to the principles outlined in the declaration of Helsinki and was approved by the ethics committee at Ruhr University Bochum (entries 3447-09 and 3483-09).

\section{Mouse models of hereditary myopathies}

Skeletal muscle samples were obtained from two published mouse models of hereditary myopathies, the MFM-filaminopathy mouse (FLNC, p.W2711X; [12]) and the mdx mouse (C57BL/10ScSn), the latter of which 
was a kind gift from Dr. Jens Schmidt (Göttingen, Germany). Littermate wildtype (WT) mouse muscles served as controls. Four (mdx model) and six (FLNC model) animals per group, respectively, were studied.

\section{Passive tension measurements}

Force measurements were done according to published protocols [51]) on isolated skinned muscle fibers from CTRL (2 subjects, 20 fibers), LGMD2A (2 subjects, 12 fibers) and MFM-filaminopathy (2 patients, 15 fibers) biopsies. Deep-frozen biopsy tissue was defrosted and skinned overnight in ice-cold low ionic-strength buffer $(75 \mathrm{mM}$ $\mathrm{KCl}, 10 \mathrm{mM}$ Tris, $2 \mathrm{mM} \mathrm{MgCl}$, $2 \mathrm{mM}$ EGTA, and $40 \mu \mathrm{g} /$ $\mathrm{ml}$ protease inhibitor leupeptin, $\mathrm{pH}$ 7.2) supplemented with 0.5\% Triton X-100. Under a binocular (Leica, Mannheim, Germany), single muscle fibers were dissected and suspended between two mini forceps attached to a piezomotor and a force transducer (Scientific Instruments, Heidelberg, Germany). Force measurements were carried out in relaxing buffer ( $8 \mathrm{mM}$ ATP, $20 \mathrm{mM}$ imidazole, $4 \mathrm{mM}$ EGTA, $12 \mathrm{mM}$ magnesium propionate, $97 \mathrm{mM}$ potassium propionate, $\mathrm{pH}$ 7.2) at room temperature. Stretching of fibers was done stepwise from slack length in 6 quick steps. Following each step the fiber was held at a constant length for $60 \mathrm{~s}$ to allow for stress relaxation. After the last step-hold, the fiber was released back to slack length. Sarcomere length (SL) was measured by laser diffraction. Passive force-SL recordings were also performed in the presence of recombinant sHSP. Briefly, the 6-ramp step stretch protocol was first carried out twice in the absence of sHSP and then repeated twice in the presence of $100 \mu \mathrm{M} \alpha \mathrm{B}$ crystallin or HSP27 (10 $\mu \mathrm{M}$ sHSP showed no effect on PT). For data analysis we considered only the peak force levels at the end of each step, which represents a mixture of elastic and viscous forces. Force was related to the crosssectional area inferred from the diameter of the specimens (at slack length), to obtain PT. Mean data points and SEM were calculated and fit with a simple polynomial. Some samples were chemically fixed (see below) immediately after mechanical measurements, usually at a stretched length, and studied for endogenous vs. exogenous sHSP localization by indirect immunofluorescence.

\section{Immunofluorescence microscopy}

Muscle biopsy samples were fixed in $4 \%$ paraformaldehyde (PFA), 15\% saturated picric acid in $100 \mathrm{mM}$ phosphate buffered saline (PBS) overnight at $4{ }^{\circ} \mathrm{C}$, dehydrated via ascending ethanol series and embedded in paraffin. Thin sections (5$7 \mu \mathrm{m}$ ) were cut with an RM 2235 Leica microtome (Mannheim, Germany). Sections were rehydrated, blocked in peroxidase blocking buffer, and a citrate-EGTA antigen recovery protocol was performed. Slides were rinsed with PBS and then blocked with $5 \%$ bovine serum albumin including $0.5 \%$ Triton X-100 for $60 \mathrm{~min}$. Subsequently, sections were incubated with primary antibodies overnight at $4{ }^{\circ} \mathrm{C}$, using one of the following antibodies against (all dilutions in PBS): HSP20 (ab125125, Abcam; 1:1000), HSP22 (ab151552, Abcam; 1:150), HSP27 (Clone 2B4, LSBio; 1:100), HSP27 (SMC 1615D12, StressMarq; 1:250), HSP27 (SR B800, MBL; 1:200), $\alpha B$-crystallin (SMC 1653A10, StressMarq; 1:250), $\alpha B$-crystallin (SR 223F, MBL; 1:400), HSP40 (Abcam, ab78437; 1:100), HSP70 (Novocastra/Leica; 1:20) HSP70 (HSPA2, Sigma HPA000798; 1:200), HSPA5 (78 kD glucose-regulated protein, Sigma, HPA038845; 1:500), Hsc70 (Heat shock cognate $71 \mathrm{kDa}$ protein, Abcam, ab2788; 1:300), HSP90 (C45G5, Cell Signaling; 1:400), HSP90 $\alpha$ (custom-made by PINEDA Berlin, 1:500), titin 9D10 PEVK (Hybridoma Bank, Iowa City, USA; 1:200), and titin custommade against PEVK C-terminal segment (Eurogentec, polyclonal, affinity-purified; 1:500). Secondary antibodies were Cy3- or FITC-conjugated IgG (Rockland; 1:400), which were incubated overnight at $4{ }^{\circ} \mathrm{C}$. For endogenous vs. exogenous HSP localization in mechanically stretched myofibers, we used a 6xHIS-conjugated IgG (Abcam ab9136; 1:400) antibody incubated overnight at $4{ }^{\circ} \mathrm{C}$. Stained samples were embedded in Mowiol supplemented with N-propyl-gallate for bleaching protection and analyzed by confocal laser scanning microscopy (Nikon, Eclipse Ti), using a $63 \times$ oil Plan-Apochromat objective.

\section{Immunoelectron microscopy}

$4 \%$ PFA fixed biopsy samples were cut into longitudinal $50-\mu \mathrm{m}$-thick sections using a VT 1000S Leica vibratome (Mannheim, Germany) and rinsed twice in PBS. Then, the muscle samples were blocked in $20 \%$ normal goat serum (NGS) for $1 \mathrm{~h}$ and incubated with primary antibodies in PBS supplemented with 5\% NGS overnight at $4{ }^{\circ} \mathrm{C}$. In addition to some of the primary antibodies used for indirect immunofluorescence (see above section), we also used anti-titin antibodies T12 (1:100; [22]) and N2A (custom-made by Eurogentec, Belgium, 1:500; [37]). The sections were then triple washed with PBS and incubated with $1.4 \mathrm{~nm}$ gold-coupled secondary antibodies (Nanoprobes, Stony Brook, NY, USA) overnight at $4{ }^{\circ} \mathrm{C}$. After extensive washing, all sections were postfixed in $1 \%$ glutaraldehyde for $10 \mathrm{~min}$ and after rinsing, sections were reacted with HQ Silver kit (Nanoprobes). After treatment with $\mathrm{OsO}_{4}$, samples were counterstained with uranyl acetate in 70\% ETOH, dehydrated and embedded in Durcupan resin (Fluka, Switzerland). Resin blocks were made and ultrathin sections prepared with a Leica Ultracut S (Mannheim, Germany) and adsorbed onto glow-discharged Formvar-carbon-coated copper grids. Microscopy was performed on a Zeiss LEO 910 electron microscope and images were taken with a TRS sharpeye CCD Camera (Troendle, Moorenwies, Germany). For some images, we measured the nearest distance across the sarcomeric Z-disc between the mean epitope positions of $\alpha \mathrm{B}$-crystallin, HSP27, HSP90, titin T12, titin 
$\mathrm{N} 2 \mathrm{~A}$, and titin PEVK antibodies using ImageJ, as described previously [38]. The distance between epitopes was plotted against SL, and data points for each antibody type were fit by two-order regression. At least 10 different cells and 30 sarcomeres per experimental condition were included in the analysis.

\section{SDS-PAGE and immunoblotting}

Deep-frozen biopsy tissue was homogenized in modified Laemmli buffer, stored on ice for $10 \mathrm{~min}$ and subsequently boiled for $10 \mathrm{~min}$ at $97{ }^{\circ} \mathrm{C}$. The protein concentration was determined by spectroscopy using Neuhoff standard protocols, SDS-PAGE was carried out using the Laemmli buffer system in slab gels containing $12.5 \%$ polyacrylamide. For immunoblot analysis the proteins were transferred onto nitrocellulose membranes by semidry electroblotting. The blots were transiently stained with Ponceau $\mathrm{S}$ to monitor transfer efficiency, then washed with Tris-buffered saline, and incubated for $2 \mathrm{~h}$ with a primary antibody. Chromogenic blotting with alkaline phosphatase conjugated secondary antibodies with nitro-blue tetrazolium and 5-bromo-4-chloro-3'-indolyphosphate was used to visualize chaperone expression on Western blots, using the following antibodies: anti-HSP27 (SR B800, MBL; concentration, 1:100), anti- $\alpha$ B-crystallin (SR 223F, MBL; 1:200), anti-HSP90 $\alpha$ (PINEDA Berlin; 1:1000), and anti- $\beta$-actin (AC-15 Sigma; 1:100). For measurements of titin:myosin heavy chain (MHC) ratio, homogenized skeletal muscle biopsy samples were analyzed by $2.5 \%$ SDS-PAGE, as described [46]. For titin phosphorylation analysis, 1.8\% SDS-PAGE was performed as described [23]. Global titin phosphorylation was determined by anti-phosphoserine/ -threonine antibodies (catalogue No. PP2551 (ECM Biosciences); Biotrend Chemicals, Cologne, Germany). To detect site-specific titin phosphorylation, we used custom-made affinity-purified phospho-serine specific antibodies against pS11878 and pS12022 in the PEVK domain of human titin (custom-made by Eurogentec, Belgium; 1:500). As secondary antibody, we used horseradish peroxidase-conjugated IgG (Acris Antibodies, Herford, Germany). For signal amplification we used the enhanced chemiluminescence Western blot detection kit (GE Healthcare). Staining was visualized using the LAS-4000 Image Reader (Fuji Science Imaging Systems) and densitometry was performed using the manufacturer's MultiGauge analysis software or ImageQuantTL (GE Healthcare). The signal on the Coomassie-stained PVDF membrane served as a means to detect total protein load (in lieu of a reference protein in the titin size range), and immunoblot signals were normalized to the corresponding PVDF signals. Finally, mean signals obtained for diseased muscle tissues were indexed to signals measured in control muscles.

\section{Statistical analysis}

Mean values of PT at a given sarcomere length were compared using two-tailed Student's t-test. Normal distribution of data was a requirement, as was the passing of the equal variance test. Mean densitometric values obtained from stained gels/Western blots were indexed to the respective mean values of human CTRL muscles and compared using Bonferroni adjusted t-test following ANOVA.

\section{Results}

Passive tension is increased in LGMD2A and MFMfilaminopathy myofibers

We measured the SL-dependent PT in skinned normal and diseased myofibers from two different MFMfilaminopathy and LGMD2A patients, respectively (Fig. 1). A relaxed preparation was stretched from slack length to a series of desired SLs (maximum, 2.8-3.0 $\mu \mathrm{m}$ ), and the peak PT in each step stretch was recorded (Fig. 1a, b). Fibers suspended in the mechanical setup showed no obvious differences in slack SL. For filaminopathy fibers we found up to $24.7 \%$ higher PT compared to CTRL fibers (Fig. 1c), for LGMD2A fibers up to $25.9 \%$ (Fig. 1d). For both groups, the difference was significant at SLs of $2.6 \mu \mathrm{m}$ and above.

\section{Titin phosphorylation is reduced in dystrophic and MFM myofibers}

Because myofiber PT is largely determined by titin, we measured titin isoform expression and phosphorylation by titin gel electrophoresis and Western blot (Fig. 2). A comparison of FLN-C, LGMD2A, and human control muscles revealed the same molecular size of titin $(\sim 3.7 \mathrm{MDa}$, Fig. 2a). Moreover, the titin:MHC ratio also remained unaltered in myopathy (Fig. 2a), suggesting that the increased PT in diseased myofibers was not due to changes in titin expression levels. In contrast, both site-specific (PEVK) phosphorylation and global titin phosphorylation were significantly reduced in the two diseased muscle groups compared to CTRL muscle (Fig. 2 b). A lower-thannormal phosphorylation state of the PEVK titin region at the two sites studied here is known to reduce titin-based PT [25]. Thus, the altered titin phosphorylation state found in the diseased muscles is unlikely to account for the PT increase of these myofibers.

\section{Heat shock proteins translocate to the titin springs in different myopathies}

Ultrastructural studies of human Vastus lateralis myocytes by electron microscopy revealed worsening of myofibrillar integrity and massive mitochondrial swelling in patient muscles, compared to normal human control muscles (Additional file 1: Figure S1a). At higher magnification, a peculiar increase in electron density was observed in the diseased samples at the sarcomeric I-bands on either side of the Z-discs, which was not seen in healthy controls. This finding implicated massive binding of proteins to the I-band, probably to the titin springs, as part of the pathology of these 

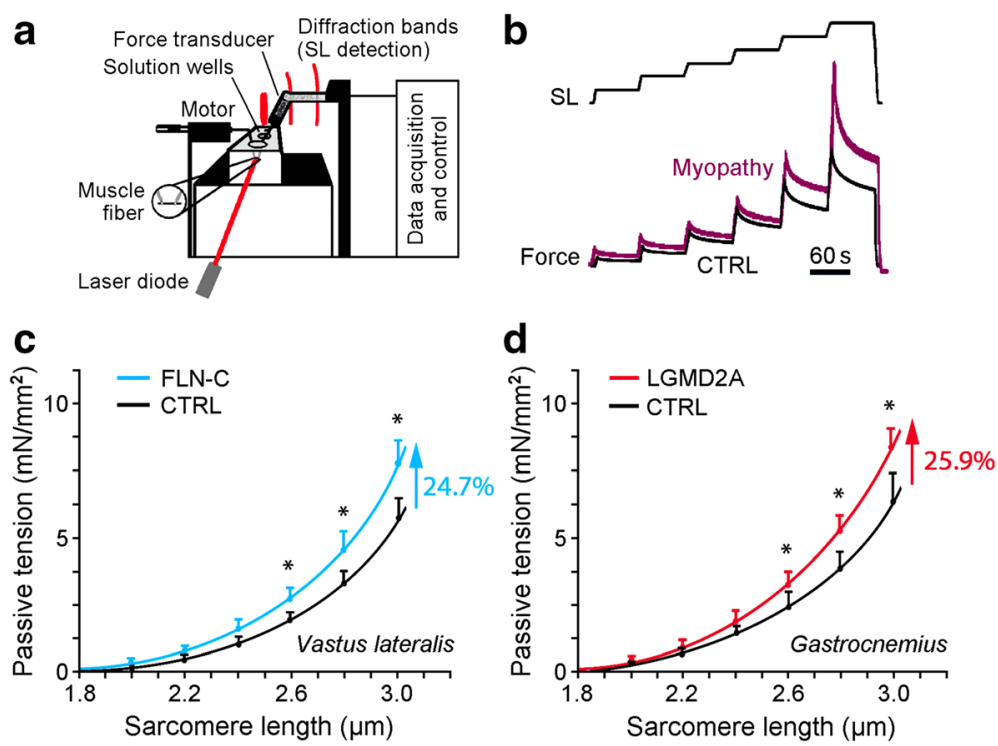

Fig. 1 Passive sarcomere length-tension relationships of isolated normal and myopathic skinned myofibers. (a) Schematic of experimental setup. (b) Mechanical measurement protocol. Sarcomere length (SL) was increased stepwise from 1.8 to 3.0 um. (c) Mean SL-dependent passive tension (PT) of normal (CTRL; $N=20$ fibers) and MFM-filaminopathy ('FLN-C'; $N=15$ fibers) vastus lateralis muscle fibers, from two different individuals/patients per group. (d) Mean PT of normal (CTRL; $N=14$ fibers) and LGMD2A ( $N=11$ fibers) gastrocnemius muscle fibers, from two different individuals/patients per group. Mean data points were fit with simple exponential functions. Symbols and error bars are means \pm SEM; ${ }^{*} p<0.05$ in Student's t-test

skeletal myopathies. We assumed that these proteins could be chaperones, such as sHSPs (HSP27, $\alpha$ B-crystallin) and HSP90, which are known to associate with I-band titin under stress $[15,31]$. The expression levels of the chaperones were measured in muscle tissue from controls and five hereditary myopathies with mutations in CAPN3 (LGMD2A), FLNC (MFM-filaminopathy), $D M D$ (Duchenne muscular dystrophy), MYOT (MFM-myotilinopathy) or VCP (IBMPFD). We found that HSP27 and $\alpha B$-crystallin were significantly increased, by a factor of $4-7$, in all myopathy types, whereas HSP90 remained unaltered within experimental error, at best showing a trend for elevated expression in myopathy (Additional file 1: Figure S1b).

Next, we performed immunocytochemical analyses using antibodies against various HSPs, in order to identify the chaperones that were translocated to the I-band region in diseased myocytes. On immunoelectron micrographs, we observed HSP20, HSPB8, HSP70 and HSC70 to be mainly at the Z-discs and in part in the cytosol (Additional file 1: Figure S2). HSP40 was found mainly in the intermyofibrillar space (presumably at the sarcoplasmic reticulum), HSPA5 consistently perinuclear, BAG3 at Z-discs in between myofibrils, and Stub1 at the Z-disc and in the Iband. Importantly, all these chaperones did not differ in their intracellular localization between CTRL, LGMD2A (Additional file 1: Figure S2) and MFM-filaminopathy (not shown). Hence, these chaperones were considered unlikely to cause the rise in titin-based PT in skeletal myopathies and not studied further.
In contrast, the sHSPs HSP27 and $\alpha \mathrm{B}$-crystallin, as well as ATP-dependent HSP90, showed strongly altered localization in diseased muscle cells (Fig. 3). Typical immunofluorescence micrographs of CTRL myocytes revealed endogenous HSP27 preferentially at the Z-discs, localizing in-between the PEVK-titin epitope (Fig. 3 a). This intracellular localization was confirmed by immuno-EM. However, in LGMD2A muscle cells, the confocal analysis suggested specific HSP27 immunoreactivity in the sarcomeric I-bands close to the PEVK epitope. Immunoelectron microscopy using anti-HSP27 antibodies showed massive labelling of the I-band on either side of the Z-disc, whereas the Z-disc itself was barely stained (Fig. 3 a). Similarly strong HSP27 signals at the elastic I-band segment were obtained in MFMfilaminopathy ('FLN-C') biopsy samples. Quantitation of the gold particle distribution indicative of HSP27 protein ( $n=30$ sarcomeres) demonstrated that $60-70 \%$ of all nanoparticles were localized to the I-band away from the Z-disc in either myopathy type (Additional file 1: Figure S3a).

Staining for endogenous $\alpha \mathrm{B}$-crystallin and counterstaining for PEVK titin in CTRL muscles revealed preferential Z-disc localization of this sHSP and additional reactivity in the myofibrillar vicinity near the Z-discs (Fig. 3b). In both LGMD2A and MFM-filaminopathy patient muscles, $\alpha B$-crystallin was at the elastic I-band region, localizing to a segment in between the Z-disc and (relatively close to) the PEVK titin epitope. Quantitation of the gold particle distribution on immunoelectron micrographs $(n=30$ sarcomeres) verified that the vast majority of $\alpha \mathrm{B}$-crystallin 

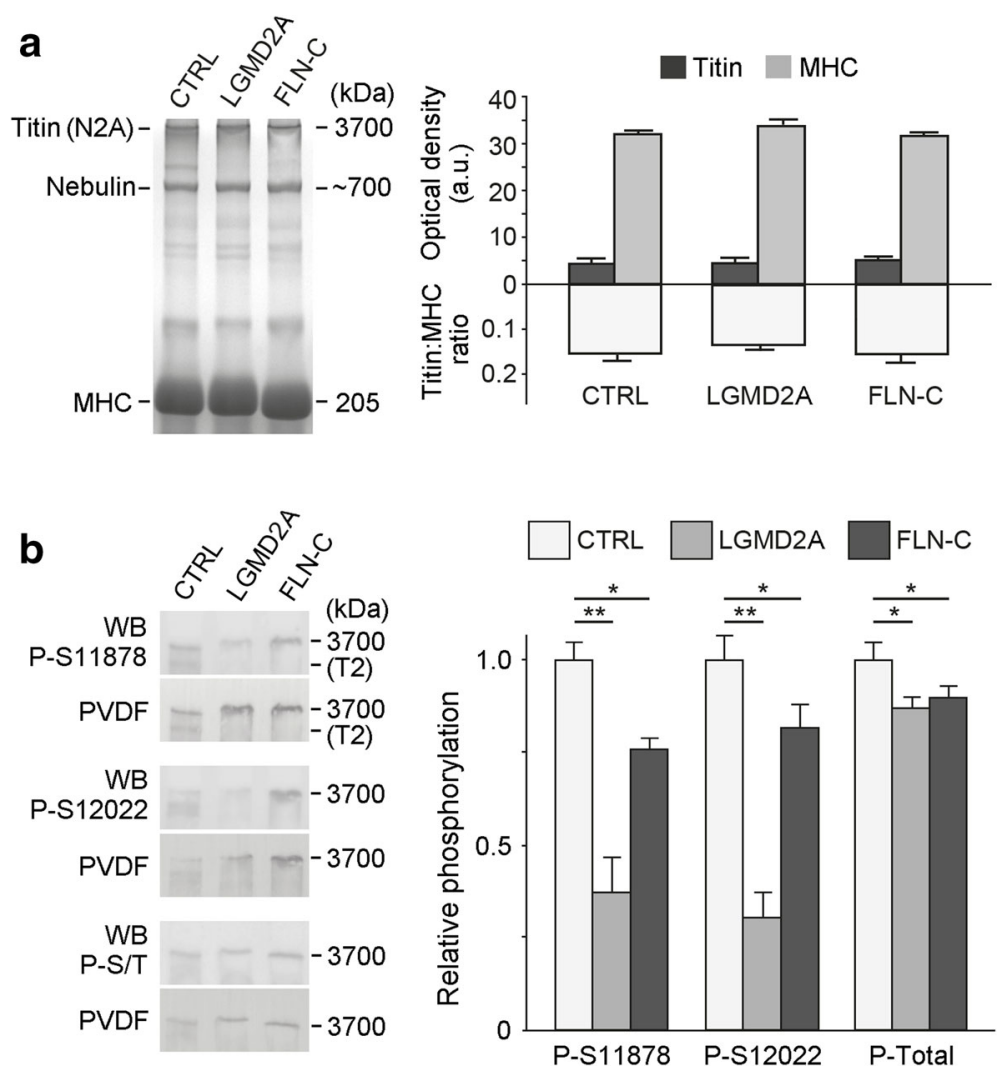

Fig. 2 Titin isoform expression and phosphorylation in normal and diseased myofibers. (a) Representative SDS-polyacrylamide gels (left panel) to monitor titin molecular size (N2A isoform) and the titin:myosin heavy chain (MHC) expression ratio in normal (CTRL), LGMD2A, and MFM-filaminopathy ('FLN-C') muscles (Vastus lateralis). Right panel shows mean titin sizes and mean titin:MHC ratios. (b) Typical Western blots of titin bands on loose SDS-polyacrylamide gels (left panel) using phosphosite-specific antibodies to phospho-serines P-S11878 and P-S12022 in the PEVK domain of titin and to all phospho-serines/-threonines in titin (P-S/T). "PVDF" is the (transiently) coomassie-stained blotting membrane, the signal from which is shown in lieu of a loading control (not available in the titin size range). Right panel shows mean phosphorylation levels for CTRL, LGMD2A and MFM-filaminopathy muscles. Data are means \pm SEM ( $n=3 /$ group); ${ }^{*} p<0.05,{ }^{* *} p<0.01$, in Bonferroni-adjusted t-test

(60-70\% of gold particles) was in the elastic I-band in myopathy fibers, presumably bound to the titin springs (Additional file 1: Figure S3 b).

Staining for endogenous HSP90 in CTRL myocytes demonstrated a broad cytosolic distribution of the chaperone, with some clustering at the sarcomeric A-bands, where it likely binds to myosin (Fig. 3c). In both LGMD2A and MFM-filaminopathy muscles, HSP90 was abundantly found at the sarcomeric I-bands very near the PEVK titin epitope. Analysis of the gold particle distribution indicative of HSP90 protein ( $\mathrm{n}=30$ sarcomeres) confirmed the preferential I-band binding ( $\sim 50 \%$ of gold particles) in myopathy and also showed additional minor associations with the Aband (25\%) and (only in filaminopathy muscles) the Z-disc (22\%) (Additional file 1: Figure S3 c).

Heat shock proteins associate with elastic titin at different I-band positions

To elucidate the precise binding sites of the three chaperones along I-band titin, we compared the SL-dependent epitope positions of anti-HSP antibodies in the sarcomere with those of different I-band titin antibodies (Fig. 4). To this end, immunoelectron microscopy was performed on CTRL, LGMD2A, and MFM-filaminopathy ('FLN-C') muscles using the T12 (start of proximal Ig-domain segment), $\mathrm{N} 2 \mathrm{~A}$, and (C-terminus of) PEVK anti-titin antibodies (Fig. 4b). In CTRL cells, HSP27 and $\alpha$ B-crystallin were localized in-between the Z-disc center and the T12 epitope (Fig. 4a, top), confirming their Z-disc association. Because of the diffuse cytosolic localization of HSP90 in CTRL cells, a distinct epitope position was not defined for this chaperone. In LGMD2A and MFM-filaminopathy myocytes, HSP27 was localized rather broadly in-between the T12 and N2A epitopes, apparently bound to the proximal/ middle titin Ig-domain segment, whereas $\alpha B$-crystallin was at a sarcomeric I-band location farther away from the Zdisc, near the N2A region (Fig. 4a). HSP90 was also localized in proximity to the N2A element, slightly closer to but never reaching the PEVK domain. Importantly, all chaperone binding sites translocated away from the Z-disc 


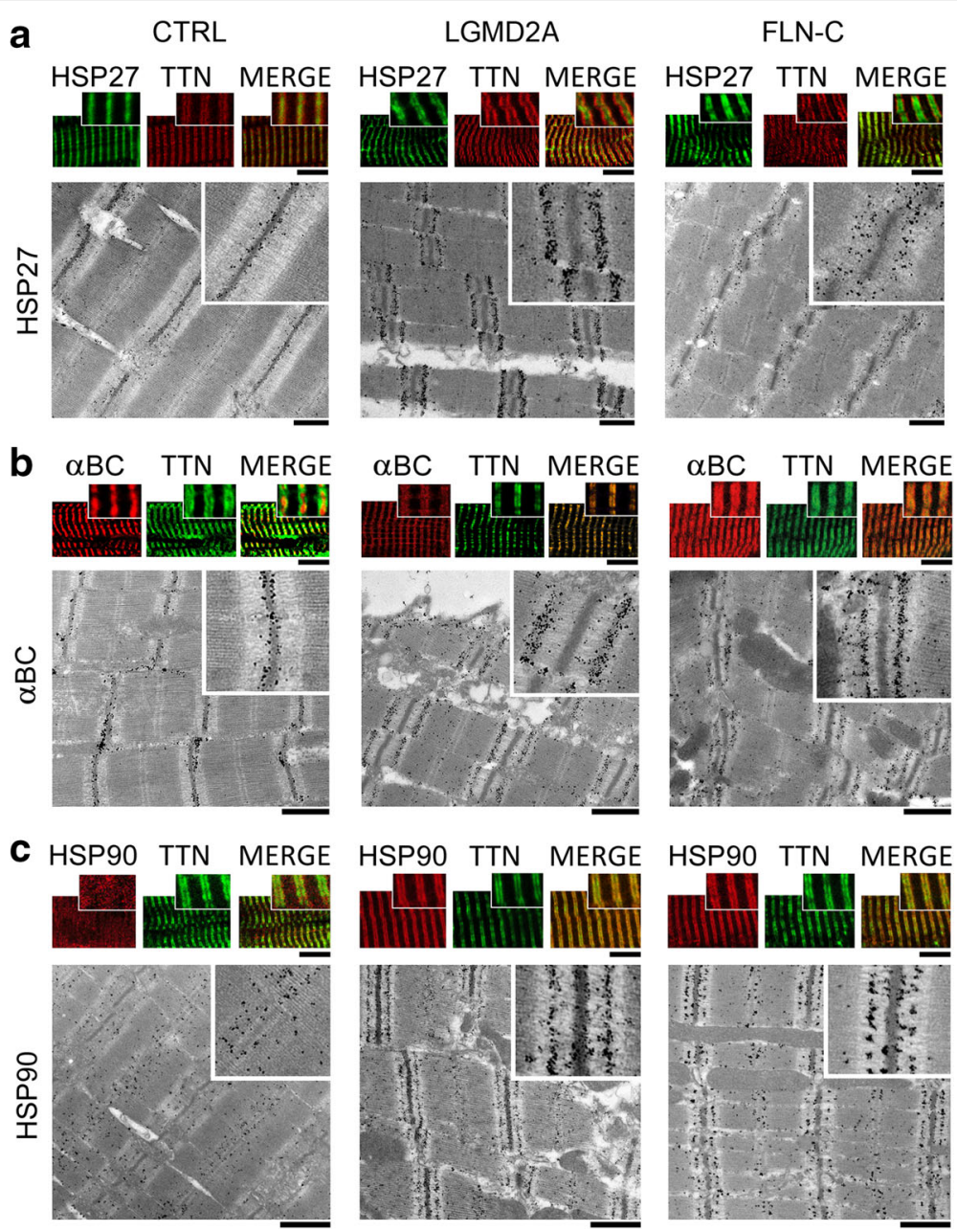

Fig. 3 Correlative immunofluorescence and immunoelectron microscopy to localize HSP27, aB-crystallin and HSP90 in normal and diseased myofibers. (a) HSP27 localization on myofibrils. Top panels: representative immunofluorescence images of CTRL, LGMD2A and MFM-filaminopathy ('FLN-C') muscle cells labeled with antibodies to HSP27 (secondary antibody: FITC-conjugated lgG). Samples were counterstained against the PEVK titin (TTN) epitope (secondary antibody: Cy3-conjugated lgG); the merged image is on the right in each group. Bottom panels show nanogold-labeled immunoelectron micrographs. Insets, higher-power images of sarcomeric regions immunostained with anti-HSP27/anti-PEVK. (b) Localization of aB-crystallin (aBC). Top panels: immunofluorescence images of myofibers labeled with anti-aB-crystallin (secondary antibody: Cy3-conjugated lgG) and counterstained for PEVK titin (secondary antibody: FITC-conjugated lgG). Bottom panels show immunoelectron micrographs, insets magnifications. (c) Localization of HSP90. Top panels: immunofluorescence images of myofibers labeled with anti-HSP90 (secondary antibody: Cy3-conjugated lgG) and counterstaining for PEVK titin (secondary antibody: FITC-conjugated lgG). Bottom panels show immunoelectron micrographs, insets magnifications. Bars, $5 \mu \mathrm{m}$ (confocal images) and $1 \mu \mathrm{m}(\mathrm{EM})$. For a quantitation of nanogold particle distribution on these and similar immunoelectron micrographs, see Additional file 1: Figure S3

with increasing SL, indicating that the HSPs bind to the elastic titin springs (and not to the thin filaments).

\section{HSP binding to elastic titin is common in hereditary myopathies, but not acquired sIBM}

We wanted to know whether the observed translocation of HSPs indicates a more general pathological response in skeletal myopathies. Hence we immunostained additional muscle biopsies from patients with other hereditary dystrophic or MFM muscle disorders, including HMERF-titinopathy, DMD, Myotilinopathy, IBMPFD due to mutation in VCP, and Desminopathy (Fig. 5a and Additional file 1: Figure S4). For comparison, we added acquired sporadic inclusion body myositis (sIBM). We found that HSP27, $\alpha \mathrm{B}$-crystallin and HSP90 were translocated to the I-band titin springs in all hereditary myopathies, with the exception of Desminopathy. In the latter, the two sHSPs were detected mainly in cytosolic aggregates, whereas HSP90 was again found in the elastic I-band region (Fig. 5a and Additional file 1: Figure S4 e). In the three different sIBM biopsies studied, none of the chaperones showed the I-band localization typical of hereditary myopathies. In these sIBM muscles, the staining pattern was similar to that in CTRL muscles, with positive immunoreactivities in the cytosol and/ or Z-disc (Fig. 5a and Additional file 1: Figure S4 f). 


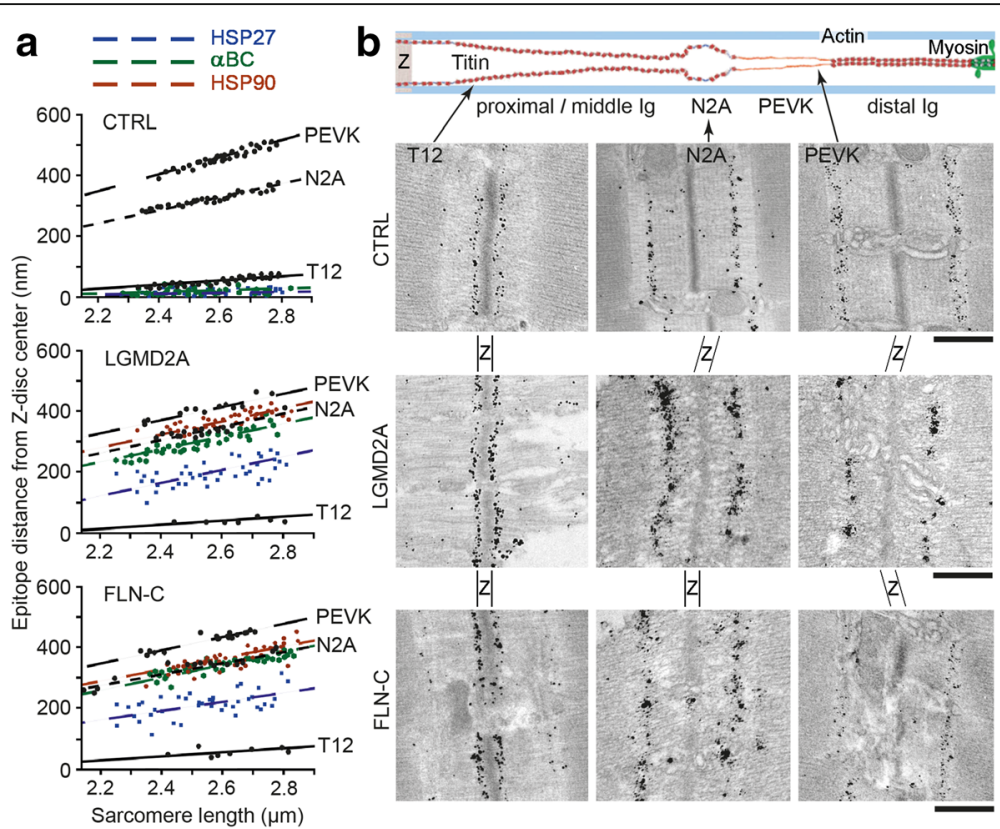

Fig. 4 Sarcomeric binding sites of chaperones relative to I-band titin epitopes. (a) Nearest epitope-to-mid-Z-disc distance of gold particles indicating the position of sarcomere-bound HSP27, aB-crystallin (aBC) and HSP90, in comparison to that of the T12, N2A and PEVK titin epitopes, on immunoelectron micrographs of CTRL, LGMD2A, and MFM-filaminopathy ('FLN-C') myofibers at different sarcomere lengths. $N=30$ sarcomeres analyzed per antibody and group. Lines are linear regression. (b) Schematic on top shows the domain architecture of I-band titin (N2A isoform) and the positions of anti-titin antibodies used (T12, N2A, PEVK). Immunoelectron micrographs below were taken from CTRL and diseased human skeletal muscle biopsy samples labeled with anti-titin T12, N2A and PEVK antibodies, respectively. Z, Z-disc region
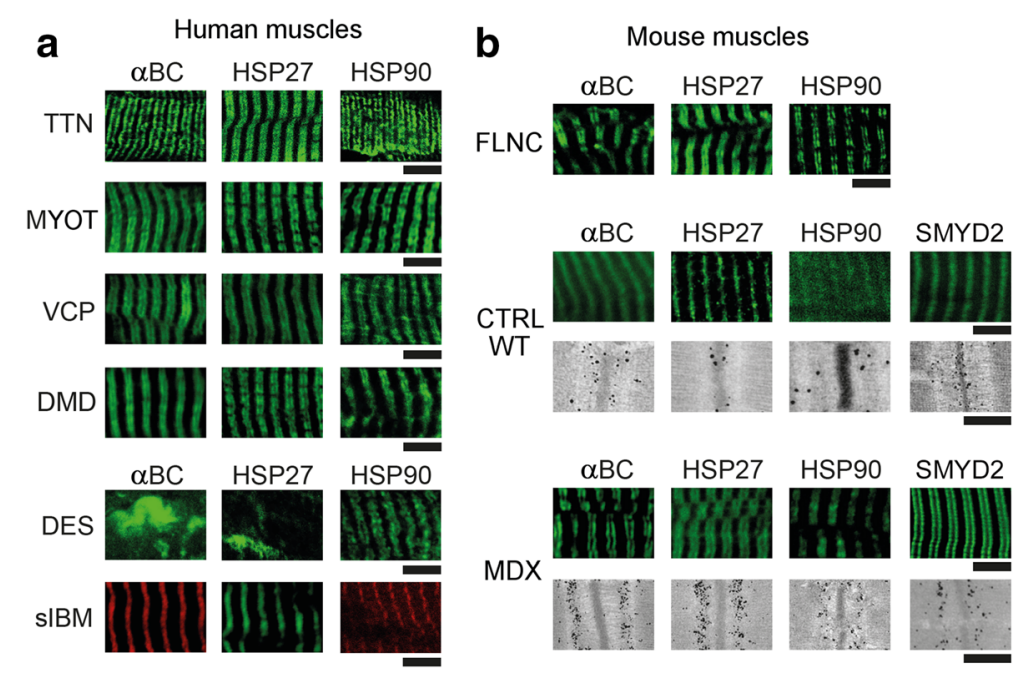

Fig. 5 Localization of sHSPs and HSP90 in myofibers of additional human myopathies and mouse models. (a) Sarcomeric I-band association of aB-crystallin (aBC), HSP27 and HSP90 in human HMERF-titinopathy (TTN), MFM-myotilinopathy (MYOT), IBMPFD due to mutations in VCP, and DMD, by indirect immunofluorescence (secondary antibodies: FITC-conjugated lgG or Cy3-conjugated lgG). In human MFM-desminopathy (DES) only HSP90 was I-band-associated, whereas the two sHSPs were found mainly in cytosolic aggregates. In sporadic inclusion body myositis (sIBM), the chaperones were detected in the cytosol and at the Z-disc (as in CTRL muscles). Note that in healthy CTRL myofibers (not shown here), the chaperones were always at the Z-disc and in the cytosol and usually did not show the doublet banding pattern typical for most myopathies. For additional immunostainings on these human samples, and counterstaining with anti-titin, see Additional file 1: Figure S4. (b) Sarcomeric 1-band association of aB-crystallin, HSP27 and HSP90 in mouse models of MFM-filaminopathy (FLNC) and Duchenne muscular dystrophy (MDX), as well as normal CTRL muscles, by indirect immunofluorescence (secondary antibodies: FITC-conjugated lgG) and nanogold-labeling immuno-EM. CTRL and MDX myofibers were also immunostained against methyltransferase Smyd2 (co-chaperone of HSP90). For additional immunostainings on these mouse samples, and counterstaining with anti-titin, see Additional file 1: Figure S5. Bars, $5 \mu \mathrm{m}$ (confocal images) and $1 \mu \mathrm{m}$ (EM) 
Taken together, translocation of HSPs to the I-bands was observed in 13 patients and 6 different types of hereditary myopathies, but not in healthy control and acquired idiopathic inflammatory myopathy muscles (Table 1).

\section{HSP binding to elastic titin is also seen in animal models of inherited myopathies}

In addition, we carried out HSP-localization experiments in two established animal models of hereditary myopathies, the dystrophic $\mathrm{mdx}$ (mutant $D M D$ ) and the FLNC (p.W2711X) MFM-filaminopathy mice, as well as in the respective littermate CTRL WT muscles. As in the human muscles, HSP27, $\alpha \mathrm{B}$-crystallin and HSP90 were translocated to the sarcomeric I-bands in both myopathy models, whereas the chaperones appeared at the Z-disc and in the cytosol in WT (Fig. 5b and Additional file 1: Figure $\mathrm{S} 5 \mathrm{a}-\mathrm{c}$ ). In an attempt to identify possible triggers for the binding of HSP90 (which has a myriad of clients) to I-band titin, we also immunostained CTRL WT and mdx mouse muscles for Smyd2, an enzyme known to methylate HSP90 as a prerequisite for the association of the Smyd2-methyl-HSP90 complex with the N2A titin region [15]. We detected Smyd2 mainly at the Z-discs and in the cytosol of WT myocytes, but at the titin springs near the PEVK epitope in mdx muscle cells, resembling the staining pattern of HSP90 (Fig. 5b and Additional file 1: Figure S5 d).

\section{Incubation of skinned human myofibers with sHSPs can increase their passive tension}

Finally, we tested whether the binding of HSPs to elastic titin could account for the increased PT seen in diseased muscle fibers (Fig. 1). Accordingly, we measured the SL-PT curve of skinned single myofibers from CTRL and LGMD2A patients in the mechanical setup, before and after incubation with HSP27 or $\alpha$ B-crystallin recombinant protein (Fig. 6a, b). Following these mechanical measurements, some fibers were prepared for indirect immunofluorescence, to measure both endogenous HSPs (Fig. 6c) and - using anti-HIS-tag antibody to recombinant, HIS-tagged $\alpha \mathrm{B}$ crystallin - exogenous $\alpha \mathrm{B}$-crystallin, the latter also in comparison to the PEVK epitope (Fig. 6d). We found that in this subset of fibers used for mechanical measurements, CTRL and LGMD2A samples showed similar amounts of endogenous HSP27 localized to the Z-disc/I-band region (Fig. 6c). In contrast, endogenous $\alpha \mathrm{B}$-crystallin was much more abundant in LGMD2A than in CTRL, localizing massively to the I-band region. Because of these differences, exogenous (recombinant) $\alpha \mathrm{B}$-crystallin could abundantly bind to the I-band region in CTRL, whereas it was barely detectable at the sarcomeres in LGMD2A (Fig. 6d). Consistent with these findings, we observed only very little effect of incubation with recombinant HSP27 on PT, in both CTRL and LGMD2A myofibers (Fig. 6b). However, incubation with $\alpha \mathrm{B}$-crystallin increased the PT of CTRL fibers on average by nearly $10 \%$, whereas the PT of LGMD2A fibers remained unaltered by this treatment (Fig. 6a). Collectively, these results suggest that sHSP-binding to elastic titin regions can increase the PT of the myofibers and explain, in part, the pathologically elevated PT of muscle cells found in LGMD2A and MFM-filaminopathy patients.

\section{Discussion}

Increased muscle stiffness is frequently seen in patients with acquired or inherited myopathies, next to muscle weakness and atrophy as the main symptoms in these disorders. Pathological increases in passive muscle stiffness were observed in DMD patients [14, 32, 33] and greatly elevated myofiber PT was reported for patients with spasticity caused by impairment of the central nervous system $[20,49]$ or patients with facio-scapulohumeral muscular dystrophy [35]. However, the mechanisms behind these alterations in passive mechanical properties have remained largely unknown. Here, we measured the PT of isolated myofibers from two groups of hereditary myopathies, LGMD2A and MFM-filaminopathy patients, and detected approximately $25 \%$ higher levels in either group compared to healthy human myofibers. We excluded titin-isoform transitions and titin phosphorylation changes as causes of this increase. Instead, we found that the PT rise in myopathic muscles is due, at least in part, to intracellular translocation of chaperones to the sarcomeric titin springs, which were devoid of chaperones in healthy muscles. The HSPs that were translocated to I-band titin in myopathy included the two sHSPs HSP27 and $\alpha B$ crystallin and the ATP-dependent chaperone HSP90. Importantly, we demonstrated that binding of these chaperones to elastic titin is common to hereditary skeletal muscle disorders, but not in acquired human sIBM. We found that the I-band titin-binding pattern of chaperones also appears in mouse models of dystrophic and MFM myopathies, but not in normal WT mouse muscles.

HSP27 and $\alpha$ B-crystallin were shown earlier to translocate to the sarcomeric Z-disc/I-band region of skeletal myofibers under stress conditions. The diverse stressors included intense exercise [50], myofibril stretching [31] or disease conditions, such as neurogenic atrophy and central core disease [18]. The cause of this translocation is incompletely understood. Potential triggers could be intracellular oxidative stress and acidosis [3,34], possibly resulting from massive mitochondrial alterations, such as those observed by us in all myopathy samples. Acidic conditions directly affect the sHSPs by promoting the formation and accumulation of large oligomers, thereby increasing chaperone activity [11, 17]. While reduced $\mathrm{pH}$ boosts the aggregation of many sHSP substrates, it also increases the SHSP-mediated protection from aggregation [3, 6]. Interestingly, acidosis raises the passive 

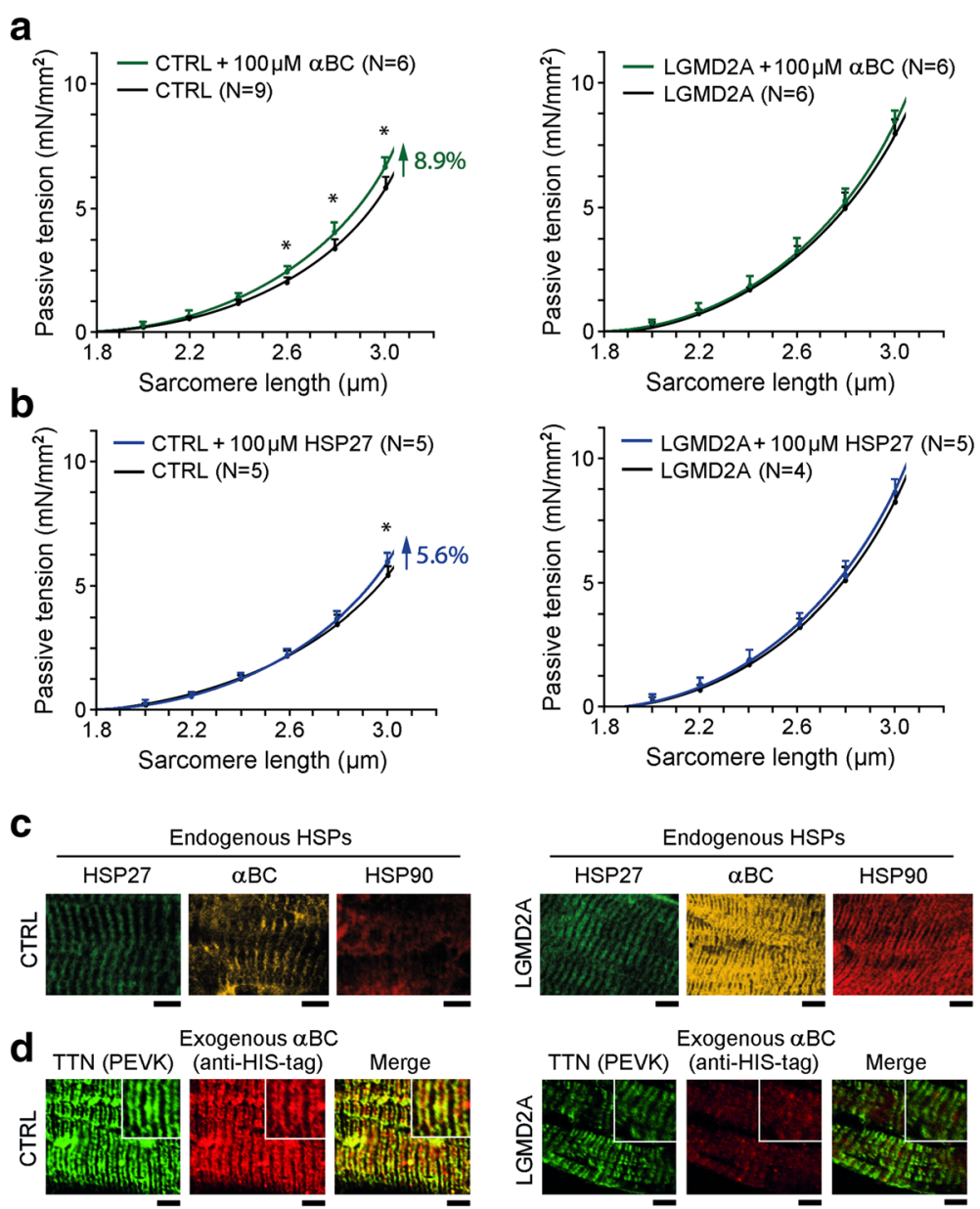

Fig. 6 Passive tension of skinned normal and myopathy myofibers in the presence of recombinant sHSPs. (a) and (b) Passive sarcomere length-tension relationships of Vastus lateralis muscle fibers from CTRL (left panels) and LGMD2A patients (right panels), before and during incubation with (a) aB-crystallin $(\mathrm{aBC})$ or (b) HSP27 recombinant protein $(100 \mu \mathrm{M})$. Data points are means \pm SEM. The number of fibers measured for each condition $(\mathrm{N})$ is indicated; fibers were obtained from 2 subjects/group. Curves are polynomial fits to the means. ${ }^{*} p<0.05$ in Student's t-test. (c) Localization of endogenous HSP27, aB-crystallin and HSP90 in skinned myofibers after force measurements, monitored by indirect immunofluorescence microscopy. Left panels, CTRL myofibers; right panels, LGMD2A myofibers. (d) Localization of exogenous (6xHIS-tagged) recombinant aB-crystallin, in relation to the PEVK titin epitope (TTN), measured using anti-6xHIS-tag Cy3-conjugated antibodies. Left panels, CTRL myofibers; right panels, LGMD2A myofibers. Insets: Higher-power images of regions-of-interest. Muscle samples were fixed in the stretched state after mechanical measurements and incubated with the respective antibodies. All bars, $5 \mu \mathrm{m}$

stiffness of skeletal muscles [44]. Although intracellular $\mathrm{pH}$ and oxidative stress were not measured in our biopsy samples, it is reasonable to speculate that exercise increases these parameters more in myopathic than in healthy muscles, which may then cause higher chaperone activity (possibly towards elastic titin) in the diseased cells.

A likely trigger for the translocation is the increased expression of sHSPs, which is typical for skeletal myocytes exposed to different stresses [30, 50]. High levels of sHSPs are beneficial, as they protect cells from oxidative stress, acidosis, energy depletion, and other unfavorable conditions [45]. In the hereditary dystrophic and MFM muscles studied by us, the expression levels of HSP27 and $\alpha$ B-crystallin were much higher than in normal control muscles. Consequently, the sarcomeres could represent a "sink" for excessive amounts of
sHSPs expressed in the diseased myocytes. A proportion of this surplus of chaperone protein may be trapped by "sticky" hydrophobic regions of the sarcomeric I-bands.

We recently showed that sarcomere stretching promotes the unfolding of titin Ig domains in the I-band [52], which results in the exposure of previously concealed hydrophobic titin regions, to which the sHSPs preferentially bind [31]. The phosphorylation state of the sHSPs, known to be relevant for their interaction with some substrates, does not seem to alter the interaction with titin domains $[19,31]$. In the LGMD2A and MFMfilaminopathy samples studied in the present work, we detected HSP27 spread out along the proximal/middle tandem-Ig segment of I-band titin. This segment contains many relatively weak domains that unfold under 
physiological stretch forces [52]. AlphaB-crystallin was found to be restricted to a narrower region near/at the N2A element of titin, which also comprises Ig domains. In contrast, the sHSP-binding spared titin's PEVK domain, a permanently unfolded (disordered) region, and the distal tandem-Ig region, which contains more stable Ig domains that rarely unfold under physiological stretch forces [52]. Assuming that sHSP-binding to the sarcomeric I-bands may be an indirect measure of the unfolded state of the titin Ig domains, our findings implicate increased unfolding of proximal/middle Ig domains in hereditary myopathy patients, possibly due to higher I-band strain than in normal myofibers. In summary, the increased association of sHSPs with the sarcomeric I-bands in myofibers of hereditary myopathy patients likely reflects increased interaction with unfolded titin Ig domains.

Conceptually, unfolding of the proximal/middle Ig domains of I-band titin raises their risk for irreversible aggregation, whereas sHSP-binding lowers this risk and protects the sarcomere [31]. Small HSPs are known to capture up to an equal weight of (partially) denatured protein before it aggregates [5]. Thus, sHSPs keep the substrate accessible to other members of the protein quality-control network, notably ATP-dependent chaperones, which are required for subsequent substrate refolding [42]. If refolding to the native state is not possible, the substrate is likely to be degraded. Hence, the binding of sHSPs to titin Ig domains could maintain the domains in a state that allows their efficient refolding. However, the binding could also be indicative of increased titin protein degradation and turnover in myopathic fibers. Either way, the sHSPs will have an important role in avoiding titin loss-of-function and preserving sarcomeric and muscle functions.

Interestingly, among the proteins overrepresented in protein aggregates of three MFM types (myotilinopathy, desminopathy and filaminopathy), there were many sarcomeric and other cytoskeletal proteins, especially Z-disc proteins, as well as various heat shock proteins (including HSP27 and $\alpha$ B-crystallin), but not titin [28, 40, 41]. In light of our results, it appears that misfolded/aberrant and potentially toxic titin is not "disposed" in aggregates, like many other cytoskeletal proteins in MFM. Instead, the sHSPs may help maintain titin in the sarcomere in a (partially) functional state, in order to preserve its role as the backbone of the sarcomere in the diseased myocyte. A deviation from this pattern of titin protection by sHSPs was observed only in the single desminopathy patient studied by us. In this biopsy sample, both HSP27 and $\alpha \mathrm{B}$-crystallin were mainly found in aggregates, the defining pathological features of this MFM. Currently we do not know why this patient muscle lacked the I-band binding pattern of sHSPs characteristic of the other myopathy types. Additional desminopathy patient samples should be studied to address this issue.
The presumed protective effect of the sHSPs on titin in most dystrophic and MFM disorders comes at the price of modestly increased passive muscle stiffness. This was suggested by the higher myofiber PT following binding of exogenous sHSPs to elastic titin in controls, but not LGMD2A fibers (which had higher PT than controls before the incubation with sHSPs). Moreover, the sHSPs can interact with and stabilize the folded Ig domains of the titin spring, which would further increase titin-based PT [9]. Because titin-dependent PT modulates the active contractile properties of skeletal myofibers [26, 36, 52], the increased PT observed in human myopathy presumably affects, to some degree, the developed tension of patient muscles. We conclude that there is a trade-off between beneficial (protection of unfolded protein) and detrimental effects (mechanical impairment) of sHSPbinding to I-band titin on sarcomere function, with consequences for overall muscle performance in myopathy.

Apart from the sHSPs, we studied a set of other chaperones for their intracellular localization in myopathic versus control muscles. However, the only chaperone that also showed a differential binding pattern was HSP90. This ATP-dependent HSP was mainly in the cytosol in controls and translocated to I-band titin in all hereditary dystrophic and MFM human samples, as well as in the DMD and MFM-filaminopathy mouse models, but not in acquired sIBM. Up to half of the cytoplasmic pool of HSP90 was associated with elastic titin (at the position of the N2A element) in human LGMD2A and MFM-filaminopathy myofibers. This binding pattern might indicate increased proteasomal degradation of Iband titin fragments. Importantly, HSP90 did not show significantly altered expression levels in the diseased muscles. Thus, a negative effect of the massive translocation of HSP90 to the titin springs could be the lack of this chaperone in myocyte compartments where it is usually required to perform its many chaperoning tasks. These findings support a concept [8] whereby the induction of HSP90 in myopathic muscles could be useful to ameliorate some pathological features in the patients.

A mechanistic link between HSP90 and sarcomeric proteins is evident from previous work. HSP90 is known as an essential modulator of myofibril organization and thick filament assembly $[4,13,24]$. Although much of the evidence is related to its association with myosin, HSP90 also binds to the N2A element of titin if methylated by a cochaperone, the methyltransferase Smyd2 [15]. The Smyd2methyl-HSP90 complex stabilizes the N2A element and helps maintain the sarcomeric Z-disc/I-band structure, which benefits muscle contraction [15, 58]. Because methylation of HSP90 by Smyd2 could be a trigger for the translocation of HSP90 to elastic titin in hereditary myopathies, we included Smyd2 in our immunoelectron/immunofluorescence microscopical analysis of WT and mdx mouse 
muscles. We found that Smyd2 translocated from the cytosol and Z-disc region in CTRL myocytes to the site of the titin N2A element in mdx, just as did HSP90. Thus, Smyd2 might guide HSP90 to the titin filaments in the sarcomeres, where the chaperone would then exert protective functions on the N2A element and possibly, other I-band proteins. In conclusion, while the functional consequences of the translocation of HSP90 to elastic titin in hereditary myopathies remain speculative, this chaperone binds to sub-sarcomeric sites other than those that associate with sHSPs, which implicates different protective functions.

\section{Conclusion}

In summary, this study used high-resolution correlative microscopy to demonstrate the sub-cellular re-distribution of members of the sHSP family and HSP90 in diseased versus healthy muscles. We provided evidence for a putative chaperone-mediated protective mechanism involved in the maintenance of the sarcomeric I-band in hereditary, but not acquired myopathies. In a panel of human muscles with dystrophic and MFM disorders due to mutations in specific genes, we found HSP27, $\alpha \mathrm{B}$-crystallin, and HSP90 to bind in a non-redundant manner to elastic titin regions, presumably to prevent unfolded titin Ig domains from aggregating and to exert additional protective functions. The interaction of sHSPs with the titin springs caused increased myofiber PT, which may be detrimental to dystrophic and MFM patients. Thus, the HSP-binding to titin is a novel phenomenon common to hereditary myopathies, which could be in place to preserve the structural integrity and function of the contractile apparatus in skeletal muscle cells.

\section{Additional file}

Additional file 1: This file contains Figures $\mathrm{S} 1$ to $\mathrm{S} 5$ and the corresponding figure legends. (PPTX $17980 \mathrm{~kb}$ )

\section{Acknowledgments}

This project was supported by the Heimer Foundation, Bielefeld, the German Duchenne Foundation, Bochum (AU, PB, RK, MV), the German Research Foundation, Research Units FOR1228 and FOR1352 (DOF), and by intramural funding (FoRUM-RUB, F857R-16 to AU, MV). HL receives funding from the Medical Research Council as part of the MRC Centre for Neuromuscular Diseases (reference G1002274, grant ID 98482) and by the European Union Seventh Framework Programme (FP7/2007-2013) under grant agreement no. 305444 (RD-Connect) and no. 305121 (NeurOmics). We thank Dr. Jens Schmidt (Göttingen, Germany) for the kind gift of the mdx mouse.

\section{Authors' contributions}

$\mathrm{AU}$ performed microscopic and mechanical measurements and wrote a manuscript draft, LB measured chaperone protein expression, PB participated in microscopic measurements, RK provided human tissue biopsies and commented on the manuscript, MvFS performed titin protein chemistry, HL provided human tissue biopsies, RS supplied both human and mouse tissue samples, DOF generated and provided the filamin-C mouse model, MV supplied human muscle biopsies and participated in manuscript writing, WAL conceived the study, participated in its design and coordination, provided supervision, and wrote the manuscript. All authors read and approved the final manuscript.

\section{Ethics approval}

All procedures performed in studies involving human participants were in accordance with the ethical standards of the institutional and/or national research committee and with the 1964 Helsinki declaration and its later amendments or comparable ethical standards.

\section{Competing interests}

The authors declare that they have no conflict of interest.

\section{Publisher's Note}

Springer Nature remains neutral with regard to jurisdictional claims in published maps and institutional affiliations.

\section{Author details}

'Department of Cardiovascular Physiology, Ruhr University Bochum, Bochum, Germany. ${ }^{2}$ Institute for Genetics of Heart Diseases, University Hospital Muenster, Muenster, Germany. ${ }^{3}$ Department of Neurology, Heimer Institute for Muscle Research, University Hospital Bergmannsheil, Ruhr University Bochum, Bochum, Germany. ${ }^{4}$ Institute of Physiology II, University of Muenster, Robert-Koch-Str. 27b, 48149 Münster, Germany. ${ }^{5}$ Institute of Genetic Medicine, Newcastle University, International Centre for Life, Central Parkway, Newcastle upon Tyne, UK. Institute of Neuropathology and Department of Neurology, University Hospital Erlangen, Erlangen, Germany. ${ }^{7}$ Institute for Cell Biology, Department of Molecular Cell Biology, University of Bonn, Bonn, Germany.

Received: 9 September 2017 Accepted: 9 September 2017

Published online: 15 September 2017

\section{References}

1. Alegre-Cebollada J, Kosuri P, Giganti D, Eckels E, Rivas-Pardo JA, Hamdani N, Warren CM, Solaro RJ, Linke WA, Fernández JM (2014) S-glutathionylation of cryptic cysteines enhances titin elasticity by blocking protein folding. Cell 156:1235-1246

2. Bär H, Fischer D, Goudeau B, Kley RA, Clemen CS, Vicart P, Herrmann H, Vorgerd M, Schröder R (2005) Pathogenic effects of a novel heterozygous R350P desmin mutation on the assembly of desmin intermediate filaments in vivo and in vitro. Hum Mol Genet 14:1251-1260

3. Barbato R, Menabò R, Dainese P, Carafoli E, Schiaffino S, Di Lisa F (1996) Binding of cytosolic proteins to myofibrils in ischemic rat hearts. Circ Res 78:821-828

4. Barral JM, Hutagalung AH, Brinker A, Hartl FU, Epstein HF (2002) Role of the myosin assembly protein UNC-45 as a molecular chaperone for myosin. Science 295:669-671

5. Basha E, O'Neill H, Vierling E (2012) Small heat shock proteins and alpha-crystallins: dynamic proteins with flexible functions. Trends Biochem Sci 37:106-117

6. Bennardini F, Wrzosek A, Chiesi M (1992) Alpha B-crystallin in cardiac tissue. Association with actin and desmin filaments. Circ Res 71:288-294

7. Bozaykut P, Ozer NK, Karademir B (2014) Regulation of protein turnover by heat shock proteins. Free Radic Biol Med 77:195-209

8. Brinkmeier H, Ohlendieck K (2014) Chaperoning heat shock proteins: proteomic analysis and relevance for normal and dystrophin-deficient muscle. Proteomics Clin Appl 8:875-895

9. Bullard B, Ferguson C, Minajeva A, Leake MC, Gautel M, Labeit D, Ding L, Labeit S, Horwitz J, Leonard KR, Linke WA (2004) Association of the chaperone alpha B-crystallin with titin in heart muscle. J Biol Chem 279:7917-7924

10. Chávez Zobel AT, Loranger A, Marceau N, Thériault JR, Lambert H, Landry J (2003) Distinct chaperone mechanisms can delay the formation of aggresomes by the myopathy-causing R120G alphaB-crystallin mutant. Hum Mol Genet 12:1609-1620

11. Chernik IS, Panasenko OO, Li Y, Marston SB, Gusev NB (2004) pH-induced changes of the structure of small heat shock proteins with molecular mass 24/27 kDa (HspB1). Biochem Biophys Res Commun 324:1199-1203

12. Chevessier F, Schuld J, Orfanos Z et al (2015) Myofibrillar instability exacerbated by acute exercise in filaminopathy. Hum Mol Genet 24:7207-7220

13. Codina M, Li J, Gutiérrez J, Kao JPY, Du SJ (2010) Loss of Smyhc1 or Hsp90a1 function results in different effects on myofibril organization in skeletal muscles of zebrafish embryos. PLoS One 5:e8416. doi:10.1371/journal.pone.0008416

14. Cornu C, Goubel F, Fardeau M (2001) Muscle and joint elastic properties during elbow flexion in Duchenne muscular dystrophy. J Physiol 533:605-616 
15. Donlin LT, Andresen C, Just S, Rudensky E, Pappas CT, Kruger M, Jacobs EY, Unger A, Zieseniss A, Dobenecker MW et al (2012) Smyd2 controls cytoplasmic lysine methylation of $\mathrm{Hsp90}$ and myofilament organization. Genes Dev 26:114-119

16. Duguez S, Bartoli M, Richard I (2006) Calpain-3: a key regulator of the sarcomere? FEBS J 273:3427-3436

17. Ehrnsperger M, Lilie H, Gaestel M, Buchner J (1999) The dynamics of Hsp25 quaternary structure. Structure and function of different oligomeric species. J Biol Chem 274:14867-14874

18. Fischer DJ, Matten J, Reimann C, Bönnemann SR (2002) Expression, localization and functional divergence of alphaB-crystallin and heat shock protein 27 in core myopathies and neurogenic atrophy. Acta Neuropathol 104:297-304

19. Frankenberg NT, Lamb GD, Overgaard K, Murphy RM, Vissing K (2014) Small heat shock proteins translocate to the cytoskeleton in human skeletal muscle following eccentric exercise independently of phosphorylation. J Appl Physiol 116:1463-1472

20. Fridén J, Lieber RL (2003) Spastic muscle cells are shorter and stiffer than normal cells. Muscle Nerve 27:157-164

21. Fürst DO, Goldfarb LG, Kley RA, Vorgerd M, Olivé C (2013) Filamin C related myopathies: pathology and mechanisms. Acta Neuropathol 125:33-46

22. Fürst DO, Osborn M, Nave R, Weber K (1988) The organization of titin filaments in the half-sarcomere revealed by monoclonal antibodies in immunoelectron microscopy: a map of ten nonrepetitive epitopes starting at the $\mathbf{Z}$ line extends close to the $M$ line. J Cell Biol 106:1563-1572

23. Hamdani N, Krysiak J, Kreusser MM, Neef S, Dos Remedios CG, Maier LS, Krüger M, Backs J, Linke WA (2013) Crucial role for $\mathrm{Ca}^{2+} /$ calmodulindependent protein kinase-II in regulating diastolic stress of normal and failing hearts via titin phosphorylation. Circ Res 112:664-674

24. Hawkins TA, Haramis AP, Etard C, Prodromou C, Vaughan CK, Ashworth R, Ray S, Behra M, Holder N, Talbot WS, Pearl LH, Strähle U, Wilson SW (2008) The ATPase-dependent chaperoning activity of Hsp90a regulates thick filament formation and integration during skeletal muscle myofibrillogenesis. Development 135:1147-1156

25. Hidalgo C, Hudson B, Bogomolovas J, Zhu Y, Anderson B, Greaser M, Labeit S, Granzier H (2009) PKC phosphorylation of titin's PEVK element: a novel and conserved pathway for modulating myocardial stiffness. Circ Res 105:631-638

26. Irving T, Wu Y, Bekyarova T, Farman GP, Fukuda N, Granzier H (2011) Thickfilament strain and interfilament spacing in passive muscle: effect of titinbased passive tension. Biophys J 100:1499-1508

27. Kaplan JC, Hamroun D (2014) The 2015 version of the gene table of monogenic neuromuscular disorders (nuclear genome). Neuromuscul Disord 24:1123-1153

28. Kley RA, Maerkens A, Leber $Y$ et al (2013) A combined laser microdissection and mass spectrometry approach reveals new disease relevant proteins accumulating in aggregates of filaminopathy patients. Mol Cell Proteomics 12:215-227

29. Kley RA, Olivé M, Schröder R (2016) New aspects of myofibrillar myopathies. Curr Opin Neurol 29:628-634

30. Kley RA, Serdaroglu-Oflazer P, Leber Y et al (2012) Pathophysiology of protein aggregation and extended phenotyping in filaminopathy. Brain 135:2642-2660

31. Kötter S, Unger A, Hamdani N, Lang P, Vorgerd M, Nagel-Steger L, Linke WA (2014) Human myocytes are protected from titin aggregation-induced stiffening by small heat shock proteins. J Cell Biol 204:187-202

32. Lacourpaille L, Gross R, Hug F, Guével A, Péréon Y, Magot A, Hogrel JY, Nordez A (2017) Effects of Duchenne muscular dystrophy on muscle stiffness and response to electrically-induced muscle contraction: a 12month follow-up. Neuromuscul Disord 27:214-220

33. Lacourpaille L, Hug F, Guével A, Péréon Y, Magot A, Hogrel JY, Nordez A (2015) Non-invasive assessment of muscle stiffness in patients with Duchenne muscular dystrophy. Muscle Nerve 51:284-286

34. Larkins NT, Murphy RM, Lamb GD (2012) Influences of temperature, oxidative stress, and phosphorylation on binding of heat shock proteins in skeletal muscle fibers. Am J Physiol Cell Physiol 303:C654-C665

35. Lassche S, Ottenheijm CA, Voermans NC, Westeneng HJ, Janssen BH, van der Maarel SM, Hopman MT, Padberg GW, Stienen GJ, van Engelen BG (2013) Determining the role of sarcomeric proteins in facioscapulohumeral muscular dystrophy: a study protocol. BMC Neurol 13:144. doi:10.1186/14712377-13-144

36. Li Y, Lang $P$, Linke WA (2016) Titin stiffness modifies the force-generating region of muscle sarcomeres. Sci Rep 6:24492. https://doi.org/10.1038/srep24492
37. Linke WA, Ivemeyer M, Olivieri N, Kolmerer B, Rüegg JC, Labeit S (1996) Towards a molecular understanding of the elasticity of titin. J Mol Biol 261:62-71

38. Linke WA, Rudy DE, Centner T, Gautel M, Witt C, Labeit S, Gregorio CC (1999) I-band titin in cardiac muscle is a three-element molecular spring and is critical for maintaining thin filament structure. J Cell Biol 146:631-644

39. Linke WA, Krüger M (2010) The giant protein titin as an integrator of myocyte signaling pathways. Physiology (Bethesda) 25:186-198

40. Maerkens A, Kley RA, Olivé M et al (2013) Differential proteomic analysis of abnormal intramyoplasmic aggregates in desminopathy. J Proteome 90:14-27

41. Maerkens A, Olivé M, Schreiner A, et al. (2016) New insights into the protein aggregation pathology in myotilinopathy by combined proteomic and immunolocalization analyses. Acta Neuropathol Commun 3;4:8. doi:10.1186/s40478-016-0280-0

42. Mchaourab HS, Godar JA, Stewart PL (2009) Structure and mechanism of protein stability sensors: chaperone activity of small heat shock proteins. Biochemistry 48:3828-3837

43. Mercuri E, Muntoni F (2013) Muscular dystrophies. Lancet 381:845-860

44. Miyake S, Ishii Y, Watari T, Huang Z, Tsuchiya T (2003) The influences of L(+ )-lactate and $\mathrm{pH}$ on contractile performance in rabbit glycerinated skeletal muscle. Jpn J Physiol 53:401-409

45. Mymrikov EV, Seit-Nebi AS, Gusev NB (2011) Large potentials of small heat shock proteins. Physiol Rev 91:1123-1159

46. Neagoe C, Kulke M, del Monte F, Gwathmey JK, de Tombe PP, Hajjar RJ, Linke WA (2002) Titin isoform switch in ischemic human heart disease. Circulation 106:1333-1341

47. Nicholls P, Bujalowski PJ, Epstein HF, Boehning DF, Barral JM, Oberhauser AF (2014) Chaperone-mediated reversible inhibition of the sarcomeric myosin power stroke. FEBS Lett 588:3977-3981

48. Ojima K, Ono Y, Hata S, Koyama S, Doi N, Sorimachi H (2005) Possible functions of p94 in connectin-mediated signaling pathways in skeletal muscle cells. J Muscle Res Cell Motil 26:409-417

49. Olsson MC, Krüger M, Meyer LH, Ahnlund L, Gransberg L, Linke WA, Larsson $L$ (2006) Fibre type-specific increase in passive muscle tension in spinal cord-injured subjects with spasticity. J Physiol 577:339-352

50. Paulsen GF, Lauritzen ML, Bayer JM, Kalhovde I, Ugelstad SG, Owe J, Bergersen HLH, Raastad T (2009) Subcellular movement and expression of HSP27, alphaB-crystallin, and HSP70 after two bouts of eccentric exercise in humans. J Appl Physiol 107:570-582

51. Prado LG, Makarenko I, Andresen C, Krüger M, Opitz CA, Linke WA (2005) Isoform diversity of giant proteins in relation to passive and active contractile properties of rabbit skeletal muscles. J Gen Physiol 126:461-480

52. Rivas-Pardo JA, Eckels EC, Popa I, Kosuri P, Linke WA, Fernández JM (2016) Work Done by Titin Protein Folding Assists Muscle Contraction. Cell Rep 14:1339-1347

53. Sanbe A, Daicho T, Mizutani R, Endo T, Miyauchi N, Yamauchi J, Tanonaka K, Glabe C, Tanoue A (2009) Protective effect of geranylgeranylacetone via enhancement of HSPB8 induction in desmin-related cardiomyopathy. PLoS One 4:e5351. https://doi.org/10.1371/journal.pone.0005351

54. Sanbe A, Yamauchi J, Miyamoto Y, Fujiwara Y, Murabe M, Tanoue A (2007) Interruption of CryAB-amyloid oligomer formation by HSP22. J Biol Chem 282:555-563

55. Srikakulam R, Winkelmann DA (2004) Chaperone-mediated folding and assembly of myosin in striated muscle. J Cell Sci 117:641-652

56. Ulbricht A, Eppler FJ, Tapia VE, van der Ven PF, Hampe N, Hersch N, Vakeel P, Stadel D, Haas A, Saftig P, Behrends C, Fürst DO, Volkmer R, Hoffmann B, Kolanus W, Höhfeld J (2013) Cellular mechanotransduction relies on tension-induced and chaperone-assisted autophagy. Curr Biol 23:430-435

57. Vissing J (2016) Limb girdle muscular dystrophies: classification, clinical spectrum and emerging therapies. Curr Opin Neurol 29:635-641

58. Voelkel T, Andresen C, Unger A, Just S, Rottbauer W, Linke WA (2013) Lysine methyltransferase Smyd2 regulates Hsp90-mediated protection of the sarcomeric titin springs and cardiac function. Biochim Biophys Acta 1833:812-822

59. Wang X, Klevitsky R, Huang W, Glasford J, Li F, Robbins J (2003) AlphaBcrystallin modulates protein aggregation of abnormal desmin. Circ Res 93:998-1005

60. Winter L, Staszewska I, Mihailovska E, Fischer I, Goldmann WH, Schröder R, Wiche $G$ (2014) Chemical chaperone ameliorates pathological protein aggregation in plectin-deficient muscle. J Clin Invest 124:1144-1157 